should be stimulated much. It is better to lower the head of the bed and give them morphin.

When the abdomen is full of blood, the bleeding to some degree has been stopped by the intra-abdominal pressure produced by the blood. Also, this intra-abdominal pressure mit. igates the shock to a great degree and the sudden relief of the pressure during the shock increases it, and for this reason we fill the abdominal cavity with salt solution before closing it and not only fill it, but by introducing the salt solution with a glass tube before the last stitches are drawn tight, thereby reestablishing the intra-abdominal pressure. The pulse is also reestablished; and by gradual absorption the physiologic effect of the salt solution is obtained.

\title{
GENERALIZED HERPES
}

\section{JAY FRANK SCHAMBERG, M.D.} PHILADELPHIA

Cases of generalized herpes zoster are of such rarity as to warrant their being recorded. But few dermatologic text-books mention generalized herpes. Within the past few years references to such cases have appeared in foreign literature. I have observed two cases in elderly men, the eruption in each instance being primarily suspected of being smallpox, although it bore greater resemblance to that of chickenpox. 'The notes of a recent case are appended:

Patient.-Mr. C., aged 66 , began to suffer pain in the left scapular region on Thursday, Nov. 25, 1909. On November 28 an extensive eruption was observed covering the left scapu-

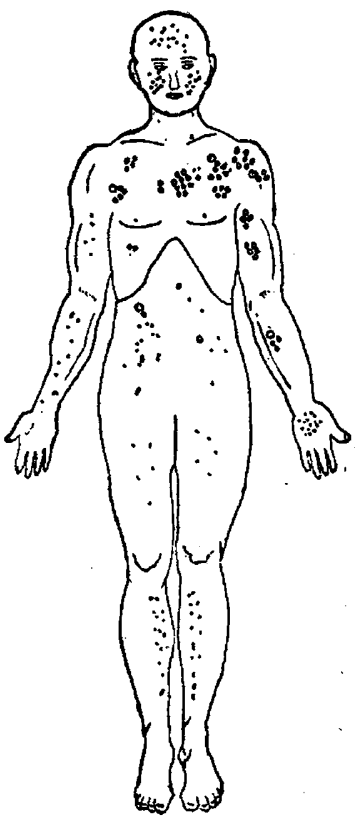

Fig. 1.--Diagram of eruption in case of generalized herpes, front view.

lar region, the left pectoral region, and the inside of the left arm. On the evening of November 29 a scattered eruption was observed by the patient on various parts of the body. The physician in attendance suspected that the case might be smallpox, and on the following day I saw the patient in consultation. The notes made at that time read as follows:

Examination.-The patient has a severe herpes zoster, the eruption of which involves the left posterior chest in the region of distribution of the thìrd dorsal nerve, and anteriorly, the left pectoral region and the inside of the left arm and hand. The lesions are large vesicles, some of which have hemorrhagic contents, and look as.if they might ultimately become necrotic.
In addition to these lesions there are scattered vesicles and small ill-defined papules on the right chest, both sides of the abdomen, the back, forearms and legs. The lesions number about 500 or more in all, and vary in size from a pin-point to a pea or larger. Most of the seattered eruptive elements are papular, but here and there distinct herpetic vesicles are seen with clear and occasionally hemorrhagic fluid. The vesicular lesions, when punctured, give exit, for the most part, to a transparent fluid. Some of the lesions are characteristically umbilicated. The palmar surface of the left hand exhibits some papules and a number of abortive vesicles. On the forehead and scalp and over the cheeks are small papular lesions. The eruption on the lower extremities is made up for the most part of scattered, barely elevated papules. The patient's temperature is practically normal; the pain has largely subsided, and there is but little itching.

The case is unquestionably one of generalized herpes. It would seem that the toxic irritant (?) responsible for the disease, instead of localizing itself as usual to one or several spinal ganglia, has exerted its effect on many nerve ganglia, thus producing a generalized eruption.

1922 Spruce Street.

\section{A CASE OF DACRYOPS FREDERICK A. KIEHLE, M.D. PORTLAND, OBE.}

Dacryops, or cystoid dilatation of a duct of the lacrimal gland, is said by all authors to be exceedingly rare. Rogman, in an exhaustive paper, succeeded in collecting only 24 cases, 2 under his own observation and 1 from the clinic of the Lawsons. ${ }^{2}$ Zur Nedden ${ }^{3}$ (1903) tabulated 33 reputed cases. It usually manifests itself to the temporal side of the median line in the vicinity of the lacrimal gland. The condition is either congenital or acquired. Removal of the entire cyst wall is indicated. Operation should preferably be from the conjunctival surface to avoid a scar, possible ptosis from injury to the levator muscle, and possible troublesome lacrimal fistula.

History.-D. D., aged 7 , was brought by her parents, Nov, 13,1909 , for advice regarding a small tumor of the left upper lid. The mother stated that the growth first became noticeable about two years ago, that it varied often from day to day, but on the whole was increasing in size. Following a protracted use of the eyes and after erying it would become much larger, sometimes nearly closing the eye. During an attack of measles it caused complete ptosis.

Examination.-The outline of a small growth slightly to the inner side of the median line with slight ptosis is noticeable on observation. On palpation it proves freely movable and painless. Upon everting the lid a bluish cyst of the size and shape of a small pecan pops down into full view, closely attached the entire length of the tarsus and apparently arising: from one of the ducts of the lacrimal gland.

Operation.-Nov. 23, 1909, under ether anesthesia the cyst was exposed to view, the adhesion to the cartilage was severed, the lacrimal duct giving origin to the cyst was tied off, and by careful blunt dissection the growth was separated from the surrounding tissue in the upper fornix. It was all but free, when the thin crypt wall being accidentally punctured, the sac collapsed and its clear, watery contents escaped. The dissection was completed and all the cyst wall removed. A single subconjunctival suture brought the tissues into proper relation and held the tarsus in position. Healing was unmarked save by a slight ecchymosis. No hyperlacrimation has appeared at any time.

814 Corbett Building.

1. Ann. d'Ophth., June, 1899

2. Tr. Ophth. Soc. U. Kingdom, xvij, 233.

3. Quoted by Fox, 1910, p. 99 